\title{
Liouville type theorem for solutions of linear partial differential equations with constant coefficients
}

\author{
by Akira Kaneko (Tokyo)
}

Pamięci przyjaźni Bogdana Ziemiana

\begin{abstract}
We discuss existence of global solutions of moderate growth to a linear partial differential equation with constant coefficients whose total symbol $P(\xi)$ has the origin as its only real zero. It is well known that for such equations, global solutions tempered in the sense of Schwartz reduce to polynomials. This is a generalization of the classical Liouville theorem in the theory of functions. In our former work we showed that for infra-exponential growth the corresponding assertion is true if and only if the complex zeros of $P(\xi)$ are absent in a strip at infinity. In this article we discuss the growth in between and present a characterization employing the space of ultradistributions corresponding to the growth.
\end{abstract}

0. Introduction. In the theory of analytic functions a well known theorem of Liouville asserts that a bounded entire function reduces to a constant. A generalized form asserts that an entire function of tempered growth (i.e. of polynomial growth) is a true polynomial. L. Schwartz $[\mathrm{S}]$ explained this theorem employing the space $\mathcal{S}^{\prime}$ of tempered distributions and suggested the following generalization:

TheOREM 0.1. Let $P(D)$ be a linear partial differential operator with constant coefficients, where $D=\left(D_{1}, \ldots, D_{n}\right), D_{j}=-i \partial / \partial x_{j}, j=1, \ldots, n$. Assume that its total symbol $P(\xi)$ has the origin as its only real zero. Then every classical solution of $P(D) u=0$, defined on the whole space $\mathbb{R}^{n}$ and of tempered growth, reduces to a polynomial. In particular, if the solution is bounded, then it reduces to a constant.

2000 Mathematics Subject Classification: 35B05, 35A27, 35E20, 32A45.

Key words and phrases: Liouville theorem, ultradistribution, infra-exponential growth, quasianalytic growth.

Partially supported by grant-in-Aid for Scientific Research (A)(2) No. 07404003. 
To illustrate our fundamental idea, we sketch the proof: $u$ can be considered as a global section of $\mathcal{S}^{\prime}$. Hence we can apply the Fourier transform to obtain

$$
P(\xi) \widehat{u}(\xi)=0 .
$$

Therefore the support of $\widehat{u}$ is contained in the set of real zeros of $P(\xi)$, which by assumption comprises the single point $\{0\}$. By the structure theorem for distributions supported by one point, we see that

$$
\widehat{u}(\xi)=Q\left(D_{\xi}\right) \delta(\xi)
$$

with a polynomial $Q$. After the inverse Fourier transform we conclude that $u(x)=Q(-x)$ is a polynomial.

The additional assertion follows from the fact that no polynomials other than constants are bounded on the whole real space.

We do not need to limit the solutions to classical ones and treat tempered distribution solutions directly. But usually we make such limitations in order to give a classical fragrance to our theorems of such type.

Schwartz's argument can be generalized without modification to the spaces of non-quasianalytic type ultradistributions or the classical solutions of the corresponding growth. This only needs preparing suitable spaces of generalized functions. We shall do it as our first task in $\S 1$. In $\S 2$, we represent the result of [Kn3] on the other extreme case, namely, the hyperfunctions, or the infra-exponential growth condition corresponding to it, where a condition on the real zeros of $P(\xi)$ at infinity becomes necessary. In $\S 3$ we discuss the most interesting case of general quasianalytic ultradistributions and the growth condition corresponding to them.

The author expresses his hearty gratitude to Dr. Yasunori Okada and Dr. G. Eysik who read the primary version of the manuscript and pointed out mistakes.

1. Case of non-quasianalytic growth. First recall the space of ultradistributions à la Komatsu $[\mathrm{Km}]$ : Let $M_{p}$ denote an increasing sequence of positive numbers satisfying the following conditions.

(M.0) extended real analyticity: for some $B, C>0$,

$$
M_{p} \geq C B^{p} p !, \quad p=0,1,2, \ldots ;
$$

(M.1) logarithmic convexity:

$$
M_{p}^{2} \leq M_{p-1} M_{p+1}, \quad p=0,1,2, \ldots ;
$$

$(M .2) \quad$ stability under convolution: for some $A, B>0$,

$$
M_{p} \leq A B^{p} \min _{0 \leq q \leq p} M_{q} M_{p-q}, \quad p=0,1,2, \ldots
$$


The condition (M.1) is equivalent to the decreasing of $M_{p} / M_{p+1}$. From this follows the inequality

$$
M_{p-q} M_{q} \leq M_{p} M_{0} \quad \text { for } 0 \leq q \leq p,
$$

hence

$$
\sum_{q=0}^{p}{ }_{p} C_{q} M_{p-q} M_{q} \leq 2^{p} M_{p} M_{0} \quad\left({ }_{p} C_{q}=\left(\begin{array}{c}
p \\
q
\end{array}\right)=\frac{p !}{q !(p-q) !}\right) .
$$

The condition (M.2) is grosso modo reciprocal to this. The following is for special use in this section:

$$
\text { non-quasianalyticity: }
$$

$$
\sum_{p=1}^{\infty} \frac{M_{p-1}}{M_{p}}<\infty .
$$

Many properties can be proved without this last assumption. For later use in $\S 3$, we try to distinguish results which really depend on (M.3).

Now for a compact set $K$, and $\mathbb{N}=\{0,1,2, \ldots\}$, we define the space of ultradifferentiable functions as follows:

$$
\begin{aligned}
\mathcal{E}^{\left\{M_{p}\right\}}(K)=\left\{\varphi(x) \in C^{\infty}(K): \exists C, h>0\right. & \forall \alpha \in \mathbb{N}^{n} \\
& \left.\left|D^{\alpha} \varphi(x)\right| \leq C h^{|\alpha|} M_{|\alpha|}\right\} .
\end{aligned}
$$

If $K$ is thin or not regular enough, we define the corresponding space to be the inductive limit of similar spaces defined on neighborhoods of $K$. Then $\mathcal{E}^{\left\{M_{p}\right\}}(K)$ becomes a DFS(= dual Fréchet-Schwartz)-type space. These define a sheaf $\mathcal{E}^{\left\{M_{p}\right\}}$ of ultradifferentiable functions of class $\left\{M_{p}\right\}$ on $\mathbb{R}^{n}$. The dual of $\mathcal{E}^{\left\{M_{p}\right\}}(K)$ is an FS(= Fréchet-Schwartz)-type space $\mathcal{E}^{\prime\left\{M_{p}\right\}}(K)$ of the corresponding ultradistributions supported by $K$. The sheaf $\mathcal{D}^{\prime\left\{M_{p}\right\}}$ of the corresponding ultradistributions is introduced via its sections which are defined as locally finite sums of ultradistributions with compact supports. As a matter of fact, under the non-quasianalyticity condition (M.3) we can define the space $\mathcal{D}^{\left\{M_{p}\right\}}(\Omega)$ of test functions with compact supports directly. But we do not use it for two reasons: first, the topology is more difficult than DFS-type; secondly, it does not work for the quasianalytic case that we employ later.

From the sequence $M_{p}$ we derive the following function of $t \geq 0$ :

$$
\mu(t)=\sup _{p} \log \frac{t^{p} M_{0}}{M_{p}} .
$$

This is a non-negative function with the following properties:

( $\mu .0)$ sublinearity: for some $B, C>0$,

$$
\mu(t) \leq B t+C
$$


$(\mu .1) \quad \mu(t)$ is a convex function of $\log t$ (i.e. $\mu\left(e^{s}\right)$ is convex in $s$ ), increasing to infinity;

( $\mu .2) \quad \mu$ and $2 \mu$ are equivalent in the sense that for some $B, C>0$,

$$
\mu(t / B)-C \leq 2 \mu(t) \leq \mu(B t)+C .
$$

The first inequality of (1.5) is rather trivial in view of the monotonicity of $\mu$. The second one corresponds to (M.2). Finally, (M.3) corresponds to the following:

( $\mu .3)$ non-quasianalyticity:

$$
\int_{1}^{\infty} \frac{\mu(t)}{t^{2}} d t<\infty
$$

Two sequences $\left\{M_{p}\right\}_{p=0}^{\infty}$ and $\left\{N_{p}\right\}_{p=0}^{\infty}$ are said to be equivalent if there exist $B, C>0$ such that

$$
C^{-1} B^{-p} M_{p} \leq N_{p} \leq C B^{p} M_{p}, \quad p=0,1,2, \ldots
$$

We shall denote by $M_{p} \equiv N_{p}$ two equivalent sequences in this sense. Equivalent sequences obviously define the same space of ultradifferentiable functions or ultradistributions.

Given a function $\mu(t)$ as above, we define conversely the sequence

$$
N_{p}=N_{0} \sup _{t>0} t^{p} e^{-\mu(t)}, \quad p=0,1,2, \ldots
$$

This again satisfies (M.0)-(M.2) if $\mu$ satisfies $(\mu .0)-(\mu .2)$, and also (M.3) if $\mu$ satisfies ( $\mu .3)$. If $\mu$ comes from $M_{p}$ via (1.4), this $N_{p}$ is equivalent to the original sequence $M_{p}$ in the above sense.

Now we introduce the Fourier type variant of Komatsu spaces. Recall that the function $\mu(t)$ characterizes the decay order of the Fourier transform for the regularity (1.3) in the Paley-Wiener type theorem. Thus we can introduce the following space of Fourier type test functions:

$$
\begin{aligned}
\mathcal{S}^{\left\{M_{p}\right\}}=\{\varphi(x) \in \mathcal{S}: \exists B, C> & 0 \forall \alpha \in \mathbb{N}^{n} \\
& \left.\left|D^{\alpha} \varphi(x)\right| \leq C B^{|\alpha|} M_{|\alpha|} e^{-\mu(|x|) / B}\right\} .
\end{aligned}
$$

This space is invariant under the Fourier transform. Hence we can define the Fourier transform on its dual space $\mathcal{S}^{\prime}\left\{M_{p}\right\}$ of " $M_{p}$-tempered" ultradistributions. Similar spaces were treated by Ehrenpreis and Gelfand-Shilov already long ago. What we remark here is that this space is localizable to the directional compactification $\mathbb{D}^{n}$ of $\mathbb{R}^{n}$ and gives a sheaf $\mathcal{S}^{\prime\left\{M_{p}\right\}}$ of " $M_{p}$-tempered" ultradistributions, whose restriction to $\mathbb{R}^{n}$ agrees with the usual sheaf $\mathcal{D}^{\prime\left\{M_{p}\right\}}$ of ultradistributions. This relation is similar to the one between the sheaf $\mathcal{Q}$ of Fourier hyperfunctions and the sheaf $\mathcal{B}$ of hyperfunctions, and contains the case $\mathcal{S}^{\prime}$ and $\mathcal{D}^{\prime}$ as a special case. Notice that in 
the precise notation of section modules in sheaf theory, $\mathcal{S}^{\prime}\left(\mathbb{D}^{n}\right)$ agrees with the usual space $S^{\prime}$ of tempered distributions, but $\mathcal{S}^{\prime}\left(\mathbb{R}^{n}\right)$ inherits no growth condition and simply reduces to $\mathcal{D}^{\prime}\left(\mathbb{R}^{n}\right)$. Later on to avoid confusion we use the symbol $\mathcal{S}^{\prime\left\{M_{p}\right\}}$ to denote the sheaf, and $\mathcal{S}^{\prime}\left\{M_{p}\right\}\left(\mathbb{D}^{n}\right)$ to denote the dual space of (1.8). The module of sections of $\mathcal{S}^{\prime\left\{M_{p}\right\}}$ supported by a compact subset $K \subset \mathbb{D}^{n}$ is denoted by $\mathcal{S}^{\prime\left\{M_{p}\right\}}[K]$ to distinguish it from the space $\mathcal{S}^{\prime\left\{M_{p}\right\}}(K)$ of all sections of $\mathcal{S}^{\prime\left\{M_{p}\right\}}$ defined on a neighborhood of $K$. If $K$ is regular enough, then $\mathcal{S}^{\prime\left\{M_{p}\right\}}[K]$ is explicitly given as the dual of $\mathcal{S}^{\left\{M_{p}\right\}}(K):=\left\{\varphi(x) \in C^{\infty}\left(K \cap \mathbb{R}^{n}\right): \exists B, C>0 \forall \alpha \in \mathbb{N}^{n}\right.$

$$
\left.\left|D^{\alpha} \varphi(x)\right| \leq C B^{|\alpha|} M_{|\alpha|} e^{-\mu(|x|) / B}\right\} .
$$

Again, the right-hand side should be replaced by an appropriate inductive limit when $K$ is thin or not regular enough. The growth condition is meaningful on a neighborhood of points at infinity contained in $K$. General sections of $\mathcal{S}^{\prime\left\{M_{p}\right\}}$ are defined via locally finite sums of such sections with "compact" supports. Notice that compact subsets of $\mathbb{D}^{n}$, when restricted to $\mathbb{R}^{n}$, are not necessarily bounded in the usual sense.

When $K$ is a compact subset of $\mathbb{R}^{n}, \mathcal{S}^{\prime}{ }^{\left\{M_{p}\right\}}[K]$ reduces to $\mathcal{D}^{\prime\left\{M_{p}\right\}}[K]$. If further $K$ is convex, the latter space can be characterized via its Fourier transform by the Paley-Wiener type theorem, as the space of entire functions $F(\zeta)$ of exponential type satisfying the estimate

$$
\forall \varepsilon>0, \exists C_{\varepsilon}>0, \quad|F(\zeta)| \leq C_{\varepsilon} e^{\varepsilon \mu(|\operatorname{Re} \zeta|)+H_{K}(\operatorname{Im} \zeta)+\varepsilon|\operatorname{Im} \zeta|} .
$$

Here $H_{K}(\eta)=\sup _{x \in K}\langle x, \eta\rangle$ denotes the supporting function of $K$. Moreover, $\mathcal{D}^{\prime\left\{M_{p}\right\}}[K]$ is a subspace of the space $\mathcal{B}[K]$ of hyperfunctions supported by $K$. The Paley-Wiener type characterization for the Fourier transform of the latter is

$$
\forall \varepsilon>0, \exists C_{\varepsilon}>0 \quad|F(\zeta)| \leq C_{\varepsilon} e^{\varepsilon|\zeta|+H_{K}(\operatorname{Im} \zeta)} .
$$

In particular, $H_{\{0\}}(\eta) \equiv 0$ if $K$ reduces to the origin. Hence the Fourier transform of $\mathcal{B}[\{0\}]$ is characterized as the space of entire functions satisfying

$$
\forall \varepsilon>0, \exists C_{\varepsilon}>0 \quad|F(\zeta)| \leq C_{\varepsilon} e^{\varepsilon|\zeta|} .
$$

This estimate is usually referred to as infra-exponential growth. This growth order is crucial throughout our theory.

The remarkable difference between the case of the pair $\mathcal{Q}-\mathcal{B}$ and of $\mathcal{S}^{\prime}-\mathcal{D}^{\prime}$ is that the restriction $\mathcal{Q}\left(\mathbb{D}^{n}\right) \rightarrow \mathcal{Q}\left(\mathbb{R}^{n}\right)=\mathcal{B}\left(\mathbb{R}^{n}\right)$ is surjective whereas the restriction $\mathcal{S}^{\prime}\left(\mathbb{D}^{n}\right) \rightarrow \mathcal{S}^{\prime}\left(\mathbb{R}^{n}\right)=\mathcal{D}^{\prime}\left(\mathbb{R}^{n}\right)$ is injective. As will be discussed in $\S 3$, the surjectivity comes from the quasianalyticity of the space of test functions. The injectivity is easily verified for general non-quasianalytic type ultradistributions, because the test functions with compact supports are dense in $\mathcal{S}\left\{M_{p}\right\}$. 
After these preparations, it is now obvious that we have the following generalization of Theorem 0.1:

Theorem 1.1. Let $P(D)$ be a linear partial differential operator with constant coefficients. Assume that its total symbol $P(\xi)$ has the origin as its only real zero. Then every classical solution of $P(D) u=0$, which is defined on the whole space $\mathbb{R}^{n}$ and has growth $O\left(e^{\varepsilon \mu(|x|)}\right)$ for any $\varepsilon>0$ for some function $\mu(t)$ satisfying $(\mu .0)-(\mu .3)$, reduces to an entire function of infra-exponential growth.

In fact, such a solution $u(x)$ can be canonically regarded as an element of $\mathcal{S}^{\prime}\left\{M_{p}\right\}\left(\mathbb{D}^{n}\right)$. Hence supp $\widehat{u}(\xi) \subset\{0\}$, and $u(x)$, as the inverse Fourier transform of a hyperfunction supported by the origin, becomes an entire function at most of infra-exponential growth. Notice that the injectivity of $\mathcal{S}^{\prime\left\{M_{p}\right\}}\left(\mathbb{D}^{n}\right) \rightarrow \mathcal{D}^{\prime\left\{M_{p}\right\}}\left(\mathbb{R}^{n}\right)$ assures that no non-trivial element of $\mathcal{S}^{\prime}\left\{M_{p}\right\}\left(\mathbb{D}^{n}\right)$ has support in the sphere at infinity $\mathbb{S}_{\infty}^{n-1}:=\mathbb{D}^{n} \backslash \mathbb{R}^{n}$. From this we see that $\operatorname{supp} \widehat{u}(\xi)$ is disjoint from $\mathbb{S}_{\infty}^{n-1}$. Except for this remark, the argument is just the same as Schwartz's original one for $\mathcal{S}^{\prime}$.

REMARK 1.1. Since $u(x)$ has the given growth $O\left(e^{\varepsilon \mu(|x|)}\right)$ for any $\varepsilon>0$ on the real axis, we can expect that the growth of $u(x)$ on the imaginary axis in the conclusion of Theorem 1.1 is in fact much more limited than the mere infra-exponential one. In the case of classical distributions or Gevrey class ultradistributions, we obtain the same growth on the imaginary axis as on the real axis, in view of the Phragmén-Lindelöf principle. For the general case it seems that a precise estimate of such type is not known yet. We leave this interesting subject for future work.

REMARK 1.2. We adopted the so called "Roumieu type" ultradistributions by the reason that their topological structure is easier, and that they are closer to the structure of Fourier hyperfunctions. For practical criterion, however, it will be better to see the growth condition of Theorem 1.1 only once, that is, for a fixed $\varepsilon>0$. This does not change the assertion essentially, because in that case we can take another, bigger $\mu(t)$ which still satisfies $(\mu .0)-(\mu .3)$ and for which the condition in the form of Theorem 1.1 is satisfied.

2. Case of infra-exponential growth. What can we expect when generalizing the growth condition from those treated in $\S 1$ ? Obviously, for solutions of exponential growth we can assert nothing from only the real zeros of $P(\zeta)$, as is easily seen by means of the exponential solutions employed in the Fundamental Principle. Thus the most general possibility is treating the solutions with infra-exponential growth:

$$
\forall \varepsilon>0, \exists C_{\varepsilon}>0 \quad|u(x)| \leq C_{\varepsilon} e^{\varepsilon|x|} .
$$


In this section we shall discuss this case. It was published in [Kn3]. We reproduce it here for completeness.

TheOREM 2.1. Let $P(D)$ be a linear partial differential operator with constant coefficients. If there exists $\delta>0$ such that $P(\zeta)$ has no complex zeros in the strip

$$
|\operatorname{Im} \zeta|<\delta,
$$

then every classical solution of $P(D) u=0$ of infra-exponential growth becomes trivial. If $P(\zeta)$ has the only real zero at the origin, and has no complex zeros in

$$
|\operatorname{Im} \zeta|<\delta, \quad|\operatorname{Re} \zeta|>\delta^{-1},
$$

then every classical solution of $P(D) u=0$ of infra-exponential growth is in fact an infra-exponential entire function.

Here, by a "classical solution of infra-exponential growth", we mean a function which, together with its derivatives up to the order of $P(D)$, is of infra-exponential growth (2.1) and satisfies the equation in the classical sense.

Pr o of. Such a solution $u$ can naturally be considered as a Fourier hyperfunction (see $[\mathrm{Kw}]$, or $[\mathrm{Kn} 2]$, Chapter 8 ). Then applying the Fourier transform we obtain $P(\xi) \widehat{u}(\xi)=0$. At finite points where $P(\xi) \neq 0$, we can multiply both sides of the above equation by the real analytic function $1 / P(\xi)$. Thus we conclude that $\widehat{u}(\xi)=0$ outside the origin. Also, in a complex neighborhood of the sphere at infinity, in view of the Seidenberg-Tarski theorem (see e.g. [H1], Appendix), we have an estimate of the form

$$
|P(\zeta)| \geq C|\zeta|^{-q},
$$

where $q>0$ is a rational number. Therefore $1 / P(\xi)$ is a multiplier for Fourier hyperfunctions in this region, and we conclude that $\widehat{u}$ is also zero there. Hence the support of $\widehat{u}$ reduces to the origin, and by the well known structure theorem for such hyperfunctions, it is of the form $J\left(D_{\xi}\right) \delta(\xi)$ with an infraexponential entire function $J(z)$. Thus we conclude that $u(x)=J(-x)$. If $P$ has no real zero even at the origin, we conclude by the same discussion that $u \equiv 0$.

We can sharpen the first assertion of the above theorem to cover solutions of exponential growth with exponential type restricted by the distance of the complex zeros of $P(\zeta)$ from the real axis:

Proposition 2.2. Assume that $P(\zeta)$ has no complex zeros in the strip $|\operatorname{Im} \zeta| \leq \delta$. Then every classical solution of growth $O\left(e^{\delta|x|}\right)$ is identically equal to 0 .

This assertion might be well known with its classical proof. We present here a proof consistent with the above discussion. Instead of Fourier hyper- 
functions, we now employ the space of Fourier ultrahyperfunctions introduced by Park-Morimoto [PM] and Sargos-Morimoto [SM]: A solution $u$ of the indicated growth has the Fourier transform which is interpreted as an element of the space $\mathcal{Q}\left(\mathbb{D}^{n}+i B_{\delta} ;\{0\}\right)$ of Fourier ultrahyperfunctions. Recall first the general notation of Fourier ultrahyperfunctions (we change the notation of $[\mathrm{SM}]$ a little in order to fit better with Sato's original notation for Fourier hyperfunctions): For two convex sets $K, L \subset \mathbb{R}^{n}$, we define the space of test functions

$$
\mathcal{P}_{*}\left(\mathbb{D}^{n}+i K ; L\right)=\bigcup_{\varepsilon>0}\left\{\varphi(z) \in \mathcal{O}\left(\mathbb{R}^{n}+i K_{\varepsilon}\right):\left|\varphi(z) e^{\varepsilon|z|+H_{L}(\operatorname{Re} z)}\right|<\infty\right\} .
$$

This is naturally endowed with the structure of DFS-type topological linear space. Its dual, denoted by $\mathcal{Q}\left(\mathbb{D}^{n}+i K ; L\right)$, is an FS-type space and is called the space of Fourier ultrahyperfunctions of growth $e^{H_{L}(x)}$ and defined on the strip $\mathbb{R}^{n}+i K$. The Fourier transform maps the space $\mathcal{P}_{*}\left(\mathbb{D}^{n}+i K ; L\right)$ isomorphically to $\mathcal{P}_{*}\left(\mathbb{D}^{n}+i L ;-K\right)$, hence $\mathcal{Q}\left(\mathbb{D}^{n}+i L ; K\right)$ to $\mathcal{Q}\left(\mathbb{D}^{n}-i K ; L\right)$. What we cited above is the special case where $K=B_{\delta}, L=\{0\}$, since we have obviously $u(x) \in \mathcal{Q}\left(\mathbb{D}^{n} ; B_{\delta}\right)$, where $B_{\delta}$ denotes the closed $\delta$-ball centered at the origin.

Finally, from $P(D) u=0$ via the Fourier transform we have $P(\zeta) \widehat{u}(\zeta)$ $=0$. Since by assumption $P(\zeta)$ has no zeros on $\mathbb{R}^{n}+i B_{\delta}$ and is bounded from below as in $(2.2)$, we conclude that $\widehat{u}(\zeta)=0$ as an element of $\mathcal{Q}\left(\mathbb{D}^{n}+\right.$ $\left.i B_{\delta} ;\{0\}\right)$, hence $u=0$.

EXAMPLE. The assumption on the complex zeros at infinity is satisfied by any hypoelliptic operator. But Theorem 2.1 covers operators which are not hypoelliptic. Notice that for the heat equation, any classical solution of growth $O\left(e^{\varepsilon|x|^{2}}\right)$ for all $\varepsilon>0$ becomes entire holomorphic. In fact, by the Tikhonov-Täcklind uniqueness theorem applied for any fixed $s \in \mathbb{R}$ such a solution agrees on $t>s$ with the one given by

$$
\int E(t-s, x-y) u(s, y) d y, \quad \text { where } \quad E(t, x)=\frac{1}{(4 \pi t)^{n / 2}} e^{-x^{2} /(4 t)} .
$$

(The analytic continuation with respect to the space variables can also be shown by the Cauchy-Kowalevsky-Zerner theorem. The analytic continuation with respect to the time variable is a property of analytic semigroups.) On the other hand, the heat equation obviously has entire solutions of order greater than 1. An example is given e.g. by the integral

$$
\int_{\mathbb{R}^{n}} e^{\xi \cdot x+|\xi|^{2} t-|\xi|^{3}} d \xi
$$

Thus our theorem asserts that for entire solutions of the heat equation, the infra-exponential growth order imposed on the real axis necessarily extends 
to the imaginary direction. This can be considered as a kind of generalization of the Phragmén-Lindelöf principle.

The above argument leaves the possibility that if the zeros of $P(\zeta)$ approach the real points at infinity more rapidly, there may be a solution of infra-exponential growth whose Fourier transform has support not only at the origin but also at these real zeros at infinity. In the latter half of this section we shall prove that this really takes place.

TheOREM 2.3. Let $P(D)$ be a linear partial differential operator with constant coefficients. Assume that for any $\delta>0$ the region

$$
|\operatorname{Im} \zeta|<\delta, \quad|\operatorname{Re} \zeta|>\delta^{-1}
$$

contains complex zeros of $P(\zeta)$. Then $P(D) u=0$ always admits a $C^{\infty}$ solution on $\mathbb{R}^{n}$ all of whose derivatives are of infra-exponential growth, but which is not entire infra-exponential.

P r o of. We first show that there exists a non-trivial Fourier hyperfunction $f(\xi)$ supported by the sphere at infinity $\mathbb{S}_{\infty}^{n-1}$ and satisfying $P(\xi) f(\xi)$ $=0$. Unfortunately we cannot present concrete examples of such $f(\xi)$. But their existence can be shown in an abstract way by observing that the kernel of the following multiplication mapping is non-trivial:

$$
P(\xi) \cdot: \mathcal{Q}\left[\mathbb{S}_{\infty}^{n-1}\right] \rightarrow \mathcal{Q}\left[\mathbb{S}_{\infty}^{n-1}\right] .
$$

Here $\mathcal{Q}\left[\mathbb{S}_{\infty}^{n-1}\right]$ denotes the space of Fourier hyperfunctions with support in $\mathbb{S}_{\infty}^{n-1}$. This is an FS-type space in good duality with the DFS-type space $\mathcal{P}_{*}\left(\mathbb{S}_{\infty}^{n-1}\right)$ of real analytic functions of exponential decay defined in a complex neighborhood of the real set $\mathbb{S}_{\infty}^{n-1}$. More concretely, in this case, an element $\varphi(\zeta)$ of $\mathcal{P}_{*}\left(\mathbb{S}_{\infty}^{n-1}\right)$ is defined in a complex domain of the form

$$
U^{\delta}:=\left\{\zeta=\xi+i \eta:|\xi|>\delta^{-1},|\eta|<\delta\right\}
$$

and there satisfies the estimate $|\varphi(\zeta)| \leq C e^{-\delta|\zeta|}$ for some $\delta>0$. For each fixed $\delta>0$, denote by $\Phi^{\delta}$ the corresponding Banach space equipped with the natural supremum norm

$$
\|\varphi\|:=\sup _{\zeta \in U^{\delta}}|\varphi(\zeta)| e^{\delta|\zeta|} .
$$

The DFS-structure of $\mathcal{P}_{*}\left(\mathbb{S}_{\infty}^{n-1}\right)$ is defined by the natural inductive limit of these $\Phi^{\delta}$ with respect to $\delta>0$. Hence by Grothendieck's theorem ([G], Chapter 4, Part 1, Section 5, Corollary 2), every bounded subset of this space is contained in some $\Phi^{\delta}$. Moreover, a bounded closed subset of $\mathcal{P}_{*}\left(\mathbb{S}_{\infty}^{n-1}\right)$ is compact and contained in some $\Phi^{\delta}$.

Now consider the mapping dual to $(2.3)$ :

$$
P(\xi) \cdot: \mathcal{P}_{*}\left(\mathbb{S}_{\infty}^{n-1}\right) \rightarrow \mathcal{P}_{*}\left(\mathbb{S}_{\infty}^{n-1}\right) .
$$


The non-triviality of (2.3) is equivalent to the non-denseness of the image $\mathcal{I}$ of this mapping. Let $\overline{\mathcal{I}}$ denote the sequential closure of $\mathcal{I}$, that is, the subspace of $\mathcal{P}_{*}\left(\mathbb{S}_{\infty}^{n-1}\right)$ obtained from $\mathcal{I}$ by adding repeatedly the limits of all sequences converging in the total space. A converging sequence in $\mathcal{P}_{*}\left(\mathbb{S}_{\infty}^{n-1}\right)$ is relatively compact, hence converges in some $\Phi^{\delta}$ in its supremum norm. Thus in view of Cantor's diagonal argument we can actually obtain $\overline{\mathcal{I}}$ by simply adding the limits of converging sequences taken from $\mathcal{I}$. We also see that any element of $\overline{\mathcal{I}}$ vanishes on the complex zero set of $P(\zeta)$ in the region $U^{\delta}$ for some $\delta>0$, which is non-void by assumption. Hence $\overline{\mathcal{I}}$ cannot be the whole space. Our assertion now follows from Lemma 2.4 below.

Now we have found an abstract element $f(\xi) \in \mathcal{Q}\left[\mathbb{S}_{\infty}^{n-1}\right]$ which satisfies $P(\xi) f(\xi)=0$ as Fourier hyperfunctions. The inverse Fourier transform $u$ of $f$ gives a solution in Fourier hyperfunctions of the equation $P(D) u=0$.

But we can say almost nothing about the nature of the inverse Fourier transform of $f$. We therefore replace this element by one with better properties. Let

$$
f(\xi)=\sum_{j=1}^{N} F_{j}\left(\xi+i \Gamma_{j} 0\right)
$$

be the boundary value representation of the Fourier hyperfunction by infraexponential defining functions $F_{j}(\zeta), j=1, \ldots, N$, on the respective wedges. By the discussion of [Kn1], we can find a positive function $\chi(t)$ of $t \geq 0$ increasing to infinity and a sequence of constants $C_{k}$ such that

$$
\left|F_{j}(\xi+i \eta)\right| \leq C_{k} e^{|\xi| / \chi(|\xi|)} \quad \text { on } 1 / k \leq|\eta| \leq \delta, j=1, \ldots, N .
$$

Then we can find an infra-exponential entire function $J(\xi+i \eta)$ with no zeros in $|\eta|<\delta$ for some $\delta>0$ and satisfying there

$$
|J(\xi+i \eta)| \geq e^{|\xi| / \chi(|\xi|)+|\xi|^{1 / 2}} .
$$

Thus $G_{j}(\zeta)=F_{j}(\zeta) / J(\zeta), j=1, \ldots, N$, are $O\left(|\zeta|^{-m}\right)$ for $m=1,2, \ldots$ uniformly on $1 / k \leq|\operatorname{Im} \zeta| \leq \delta$ for $k=1,2, \ldots$ Now the new Fourier hyperfunction $g(\xi)=f(\xi) / J(\xi)$, or more precisely the one defined by $G_{j}(\zeta)$, $j=1, \ldots, N$, again has support in $\mathbb{S}_{\infty}^{n-1}$ and satisfies $P(\xi) g(\xi)=0$.

The inverse Fourier transform $v$ of $g$ is a Fourier hyperfunction satisfying $P(D) v=0$. Moreover, by the above estimate for the defining functions of $g, v$ can be directly calculated by the integral

$$
v(x)=\sum_{j=1}^{N}(2 \pi)^{-n} \int_{\mathbb{R}^{n}} G_{j}\left(\xi+i \varepsilon \eta_{j}\right) e^{i x\left(\xi+i \varepsilon \eta_{j}\right)} d \xi,
$$

where $\eta_{j} \in \Gamma_{j}, j=1, \ldots, N$, are unit vectors. This gives a continuous function of growth $e^{\varepsilon|x|}$. Since the integral is independent of the choice of 
$\varepsilon>0, v(x)$ is actually of infra-exponential growth. The same holds for all finite order derivatives of $v$. This proves our assertion.

The following lemma, which we used in the above proof, may be well known. Since we could not find suitable references just containing it as it is, we give a simple proof for the convenience of the reader.

Lemma 2.4. A convex subset $H$ of a DFS-space $E=\lim _{k} B_{k}$ is closed if and only if it is sequentially closed.

Proof. By Banach-Dieudonné's theorem $H$ is closed if and only if for any weakly closed equicontinuous subset $K \subset E, H \cap K$ is weakly closed (see [G], Chapter 4, Part 2, Section 3, Corollary 1). Notice that in a DFSspace the notions of weakly bounded, strongly bounded, weakly relatively compact, strongly relatively compact and equicontinuous are all equivalent. Let $K$ be such a set which is weakly closed, and let $L$ be its closed convex hull in the strong sense. It is bounded, hence compact and contained in some of the defining Banach spaces $B_{k}$ of $E$. Since $H$ is sequentially closed, $H \cap L$ is closed and compact in $B_{k}$, hence in $E$. Since for convex sets the notions of weak and strong closedness agree, $H \cap L$ is weakly closed. Thus $H \cap K=(H \cap L) \cap K$ should also be weakly closed.

REMARK 2.1. In the above proof of Theorem 2.3, we gave a solution of infra-exponential growth with $C^{\infty}$ regularity. But we can always exhibit an entire analytic solution. To show this, we replace the entire infra-exponential function $J(\zeta)$, employed to make $g$ rapidly decreasing, by the entire function $e^{\varepsilon \zeta^{2}}$. It is obvious that $g(\xi)=f(\xi) e^{-\varepsilon \xi^{2}}$ has the entire function $v$ as its inverse Fourier image, as is seen from formula (2.6). It is also obvious that $v$ satisfies $P(D) v=0$. But we have to check that $v \not \equiv 0$, or equivalently $g \not \equiv 0$ as a Fourier hyperfunction. This can be proved by the Phragmén-Lindelöf principle as in Palamodov [P] (cf. Lemma 3.3 below). Here for our purpose it suffices to see that this holds at least for some $\varepsilon>0$, which can be shown more easily: Set $g_{\varepsilon}(\xi)=f(\xi) e^{-\varepsilon \xi^{2}}$ and assume that these are equal to zero as Fourier hyperfunctions for all $\varepsilon>0$. But as $\varepsilon \rightarrow 0$ we have $g_{\varepsilon} \rightarrow f$ as Fourier hyperfunctions. In fact, take a test function $\varphi \in \mathcal{P}_{*}\left(\mathbb{S}_{\infty}^{n-1}\right)$. Then we have

$$
\left\langle g_{\varepsilon}, \varphi\right\rangle=\left\langle f, \varphi e^{-\varepsilon \xi^{2}}\right\rangle .
$$

Here if $\varphi$ belongs to the Banach space $\Phi^{\delta}$ in the notation used in the proof of Theorem 2.3, then as $\varepsilon \rightarrow 0$ we have

$$
\sup _{\zeta \in U^{\delta / 2}}\left|\varphi(\zeta)\left(1-e^{-\varepsilon \zeta^{2}}\right)\right| e^{(\delta / 2)|\zeta|} \rightarrow 0,
$$

hence $\varphi(\xi) e^{-\varepsilon \xi^{2}} \rightarrow \varphi$ in $\Phi^{\delta / 2}$, hence in $\mathcal{P}_{*}\left(\mathbb{S}_{\infty}^{n-1}\right)$. Thus $(2.7)$ tends to $\langle f, \varphi\rangle$. Therefore $g_{\varepsilon} \rightarrow f$ (weakly, hence strongly, because $\mathcal{Q}\left[\mathbb{S}_{\infty}^{n-1}\right]$ is an FS-space). Thus, given that $f \neq 0$, some of $g_{\varepsilon}$ should be non-trivial. 
Notice, however, that the growth order of such entire solutions $u$ in the imaginary direction is very high in general.

One might think that the discussion of Theorem 2.3 together with the use of modified Fourier hyperfunctions would give similar counter-examples even for hypoelliptic operators which are non-elliptic. But the inverse Fourier image of a modified Fourier hyperfunction calculated by the modified version of formula (2.6) need not be of infra-exponential growth on the real axis.

3. Case of quasianalytic growth. Now we consider the case of quasianalytic type ultradistributions or the growth order corresponding to them. The phenomenon is most sophisticated and interesting in this case. Again, we need to prepare the appropriate spaces. This time we replace the conditions $(M .3),(\mu .3)$ of non-quasianalyticity employed in $\S 1$ by the corresponding conditions of quasianalyticity:

quasianalyticity:

$$
\sum_{p=1}^{\infty} \frac{M_{p-1}}{M_{p}}=\infty
$$

( $\mu .4)$ quasianalyticity:

$$
\int_{1}^{\infty} \frac{\mu(t)}{t^{2}} d t=\infty .
$$

We define the space and sheaf $\mathcal{E}^{\left\{M_{p}\right\}}$ of quasianalytic type ultradifferentiable functions, the corresponding space and sheaf $\mathcal{D}^{\prime\left\{M_{p}\right\}}$ of ultradistributions, replacing (M.3) and ( $\mu .3)$ by $(M .4)$ and $(\mu .4)$, respectively. For the general discussion see de Roever [dR]. We can also introduce the associated Fourier analogue $\mathcal{S}^{\left\{M_{p}\right\}}, \mathcal{S}^{\prime\left\{M_{p}\right\}}$ just in the same way as in $\S 1$.

We first examine the existence of the obstruction at infinity similar to the case of Fourier hyperfunctions or of infra-exponential growth.

Lemma 3.1. Let $M_{p}$ be a quasianalytic weight sequence. Then the restriction mapping $\mathcal{S}^{\prime\left\{M_{p}\right\}}\left(\mathbb{D}^{n}\right) \rightarrow \mathcal{D}^{\prime\left\{M_{p}\right\}}\left(\mathbb{R}^{n}\right)$ is surjective.

This comes from the flabbiness of the sheaf $\mathcal{S}^{\prime\left\{M_{p}\right\}}$ of quasianalytic type Fourier ultradistributions. In [H2] Hörmander first remarked that the flabbiness, which was considered to be the most distinguished property of the sheaf $\mathcal{B}$ of hyperfunctions, in fact also holds for the sheaf $\mathcal{D}^{\left\{M_{p}\right\}}$ of any quasianalytic type ultradistributions, and showed that it is a property dual to quasianalyticity. His proof follows Martineau's proof of the flabbiness of $\mathcal{B}$, and it just as well applies to our sheaf $\mathcal{S}^{\prime}\left\{M_{p}\right\}$ of quasianalytic type Fourier ultradistributions. Actually the proof of the flabbiness of $\mathcal{Q}$ given in [Kn2] goes along the same lines. 
The essence of the proof is the following: Given $f \in \mathcal{D}^{\prime\left\{M_{p}\right\}}\left(\mathbb{R}^{n}\right)$, we first decompose it into a locally finite sum with compact supports $K_{\lambda}$ of sections $f_{\lambda}$ of $\mathcal{D}^{\prime\left\{M_{p}\right\}}$, hence of $\mathcal{S}^{\prime}\left\{M_{p}\right\}$. From the quasianalyticity of the test functions, we can see at once that the inclusion $\mathcal{S}^{\left\{M_{p}\right\}}(K)^{\prime} \rightarrow \mathcal{S}^{\left\{M_{p}\right\}}(L)^{\prime}$ has dense image for two connected compact sets $K \subset L$. Employing this fact, we add up $f_{\lambda}$ in $\mathcal{S}^{\prime\left\{M_{p}\right\}}\left(\mathbb{D}^{n}\right)$ modifying them by elements with supports in the farthest part of the boundary of $K_{\lambda}$ in such a way that $f_{\lambda}$ converges in $\mathcal{S}^{\prime\left\{M_{p}\right\}}\left(\mathbb{D}^{n}\right)$. We push forward the modification successively to the adjacent farther sets and let the tail of the series converge in $\mathcal{S}^{\left\{M_{p}\right\}}(\{R \leq|x| \leq \infty\})^{\prime}$ for each $R>0$. In this way we obtain an element $g$ of $\mathcal{S}^{\prime\left\{M_{p}\right\}}\left(\mathbb{D}^{n}\right)$ which agrees with the given $f$ on $\mathbb{R}^{n}$.

Lemma 3.2. Let $M_{p}$ be a quasianalytic weight sequence. Then there exists a non-trivial element of $\mathcal{S}^{\prime\left\{M_{p}\right\}}\left(\mathbb{D}^{n}\right)$ whose support is in $\mathbb{S}_{\infty}^{n-1}$.

This is shown just in the same way as for the Fourier hyperfunctions. Take, e.g., the function $e^{x}$ of one variable. It is a section of $\mathcal{D}^{\prime\left\{M_{p}\right\}}(\mathbb{R})$, hence it can be extended to an element $f$ of $\mathcal{S}^{\prime\left\{M_{p}\right\}}(\mathbb{D})$ by the above lemma. Then

$$
g=(d / d x-1) f
$$

is the desired element of $\mathcal{S}^{\prime}{ }^{\left\{M_{p}\right\}}(\mathbb{D})$. Actually, it is clear that it vanishes on $\mathbb{R}$. (One cannot, however, verify it via test functions directly, because we have no test functions with compact support! We may, e.g., employ the inclusion $\mathcal{A} \rightarrow \mathcal{S}^{\left\{M_{p}\right\}} \rightarrow \mathcal{S}^{\prime\left\{M_{p}\right\}}$ with compatibility of calculus rule.) On the other hand, it cannot be trivial, because if so, we would have $f=0$ via the Fourier transform and division by $i \xi-1$. Thus $g$ has non-trivial support contained in $\mathbb{S}_{\infty}^{0}=\{ \pm \infty\}$. An easy check shows that $-\infty$ is not in $\operatorname{supp} g$. Thus we found an element of $\mathcal{S}^{\prime}\left\{M_{p}\right\}(\mathbb{D})$ with one-point support. (We can construct a more concrete example modifying the technique of Morimoto-Yoshino [MY] for the case of Fourier hyperfunctions. This will be discussed in our forthcoming paper.)

An example in general dimension can be $g\left(x_{1}\right) \delta\left(x^{\prime}\right)$ with $g$ as above. Its meaning should be obvious:

$$
\left\langle g\left(x_{1}\right) \delta\left(x^{\prime}\right), \varphi(x)\right\rangle=\left\langle g\left(x_{1}\right), \varphi\left(x_{1}, 0\right)\right\rangle .
$$

Notice, however, that the product expression $g\left(x_{1}\right) h\left(x^{\prime}\right)$ is not generally legitimate, because the space $\mathbb{D}^{n}$ does not have the corresponding product structure.

The following is essentially the three-line theorem, and in fact, in the case of one variable, the last assertion, which we need in the sequel, can be directly obtained from the three-line theorem by applying it to the defining function of the Fourier hyperfunction. Hence our assertion may be consid- 
ered as a generalization of the three-line theorem to several variables. Cf. Palamodov $[\mathrm{P}]$, where the same bound is introduced for a more delicate aspect of weighted Fourier hyperfunctions.

LEMmA 3.3. Let $\mu(t)$ be an increasing function which grows at least linearly. Assume that for some $C, A>0$,

$$
\mu(t) \leq C e^{A t} .
$$

Then the space $\mathcal{P}_{*}^{\mu}\left(\mathbb{S}_{\infty}^{n-1}\right)$ of real analytic functions each of which, for some $\delta>0$, is defined in $U^{\delta}$ and satisfies the decay condition

$$
|\varphi(\zeta)| \leq C e^{-\delta \mu(|\zeta|)},
$$

is dense in the space $\mathcal{P}_{*}\left(\mathbb{S}_{\infty}^{n-1}\right)$. Equivalently, the dual space $\mathcal{Q}^{\mu}\left[\mathbb{S}_{\infty}^{n-1}\right]$ contains $\mathcal{Q}\left[\mathbb{S}_{\infty}^{n-1}\right]$ injectively.

Proof. Let $\varphi \in \mathcal{P}_{*}\left(\mathbb{S}_{\infty}^{n-1}\right)$ be any element. Assume that $\varphi \in \Phi^{\delta}$, that is, $\varphi$ is holomorphic and satisfies the estimate $|\varphi(\zeta)| \leq C e^{-\delta|\zeta|}$ on $U^{\delta}$. Set

$$
\varphi_{\varepsilon}:=\exp \left\{-\varepsilon \cosh A^{\prime} \zeta\right\} \varphi(\zeta) .
$$

Then if $A^{\prime}>A$ and $A^{\prime} \delta<\pi / 2$ we have obviously $\varphi_{\varepsilon} \in \mathcal{P}_{*}^{\mu}\left(\mathbb{S}_{\infty}^{n-1}\right)$, and as $\varepsilon \downarrow 0$ it converges to $\varphi$ in $\Phi^{\delta / 2}$, hence in $\mathcal{P}_{*}\left(\mathbb{S}_{\infty}^{n-1}\right)$. Since we can choose $\delta>0$ as small as we like, we conclude that any $\varphi$ can be approximated by a sequence from $\mathcal{P}_{*}^{\mu}\left(\mathbb{S}_{\infty}^{n-1}\right)$.

After these preparations, we can now discuss the existence of solutions tempered in the present sense. Unfortunately, our result is still partial, which is expressed by the following analogue of Liouville's theorem:

TheOREM 3.4. Let $P(D)$ be a linear partial differential operator with constant coefficients. Assume that $P(\xi)$ has only the origin as a real zero and that there exist positive constants $A$, a with $0<a \leq 1$ such that $P(\zeta)$ has no complex zeros in

$$
|\operatorname{Im} \zeta| \leq A|\operatorname{Re} \zeta|^{-a}, \quad|\operatorname{Re} \zeta| \geq 1 / A .
$$

Then every classical solution of $P(D) u=0$, globally defined on $\mathbb{R}^{n}$ and of growth $O\left(e^{\varepsilon|x| /(\log |x|)^{a}}\right)$ for any $\varepsilon>0$, is in fact entire infra-exponential. If furthermore $P(\xi)$ has no real zero, then $u \equiv 0$. Here again the growth is understood to be imposed on the derivatives of u up to the order of $P(D)$.

P r o of. First notice that the weight function

$$
t /(\log t)^{a}, \quad t \geq 3,
$$

corresponds to

$$
M_{p}=p^{p}(\log p)^{a p}, \quad p \geq 3 .
$$


In fact, take (3.2). If we set $s=t /(\log t)^{a}$, then for large $t$ we have $C^{-1} t^{1 / 2} \leq$ $s \leq C t$ with some $C>0$, hence $t=s(\log t)^{a}$ satisfies

$$
\frac{1}{C} s(\log s)^{a} \leq t \leq C s(\log s)^{a}
$$

with another $C>0$. In

$$
N_{p}=\sup _{t>0} t^{p} e^{-A t /(\log t)^{a}} \equiv \sup _{s>0}\left\{s(\log s)^{a}\right\}^{p} e^{-A s},
$$

the maximum of the last quantity is taken at $s$ satisfying

$$
\left(1+\frac{a}{\log s}\right) p-A s=0,
$$

which is in the region $s \leq 2 p / A$ for large $p$. But in this region we have

$$
\left\{s(\log s)^{a}\right\}^{p} e^{-A s} \leq\left(\frac{2 p}{A}\right)^{p}\left(\log \frac{2 p}{A}\right)^{a p} e^{-2 p} \equiv p^{p}(\log p)^{a p},
$$

and an equivalent value is actually attained at $s=2 p / A$. Conversely, assume (3.3). In

$$
\nu(t)=\sup _{p} \log \frac{t^{p}}{p^{p}(\log p)^{a p}}=\sup _{p}\{p \log t-p \log p-a p \log \log p\},
$$

the supremum for continuous $p$ is attained at $p$ satisfying

$$
\log t-\log p-1-a \log \log p-\frac{a}{\log p}=0,
$$

that is,

$$
t / e=p(\log p)^{a} e^{a / \log p} \sim p(\log p)^{a},
$$

which obviously lies in $(t / e)^{1 / 2} \leq p \leq t / e$, hence in

$$
\frac{1}{C} \frac{t}{(\log t)^{a}} \leq p \leq C \frac{t}{(\log t)^{a}}
$$

for some $C>0$. In this region we have

$$
\frac{t}{p(\log p)^{a}} \sim e,
$$

hence $\nu(t) \sim p$ is equivalent to (3.2).

By assumption, $P(\xi)$ is invertible on the real axis as a function. We shall show that the inverse $1 / P(\xi)$ serves as a well defined multiplication operator from $\mathcal{S}^{\prime}\left\{M_{p}\right\}\left[\mathbb{S}_{\infty}^{n-1}\right]$ to $\mathcal{Q}^{\mu}\left[\mathbb{S}_{\infty}^{n-1}\right]$ with $\mu(t)=e^{A t}$. In view of the Leibniz rule and (1.2), it suffices to see that for any $\varepsilon>0$ we have, with some $B_{\varepsilon}, C_{\varepsilon}>0$,

$$
\left|\partial_{\xi}^{\alpha} \frac{1}{P(\xi)}\right| \leq C_{\varepsilon} B_{\varepsilon}^{|\alpha|} M_{|\alpha|} \exp \left\{\varepsilon e^{A|\xi|}\right\} \quad \text { for } \alpha \in \mathbb{N}^{n} .
$$

Notice first that by Seidenberg-Tarski's theorem as employed in the proof of Theorem 2.1, there exists a fixed constant $k_{0}$ such that $|P(\zeta)| \geq C|\xi|^{-k_{0}}$ in 
the region (3.1). By the assumption on the zeros of $P(\zeta)$, we can apply the Cauchy integral formula for a polydisc with center at a real point $\xi$ which is far enough from the origin and with the radii $\delta=A|\xi|^{-a}$ so that we obtain

$$
\left|\partial_{\xi}^{\alpha} \frac{1}{P(\xi)}\right| \leq C A^{-|\alpha|}|\xi|^{a|\alpha|+k_{0}}|\alpha| !
$$

Set $k=|\alpha|, t=|\xi|$. Then we have, with suitable constants,

$$
\begin{aligned}
\sup _{\xi}|\xi|^{a|\alpha|+k_{0}} A^{-|\alpha|}|\alpha| ! \exp \left\{-\varepsilon e^{A|\xi|}\right\} & =\sup _{t>0} t^{a k+k_{0}} A^{-k} k ! \exp \left\{-\varepsilon e^{A t}\right\} \\
& =A^{-k} k ! \sup _{s>0}\left(\frac{1}{A} \log \frac{s}{\varepsilon}\right)^{a k+k_{0}} e^{-s} \\
& \leq C B^{k} k^{k}(\log k)^{a k},
\end{aligned}
$$

whence (3.4) follows. Thus we have a diagram

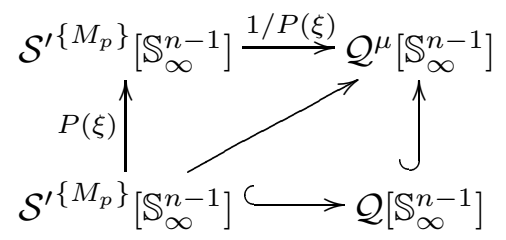

The commutativity of this diagram is easily verified via the inner product with a test function from $\mathcal{P}_{*}^{\mu}\left(\mathbb{S}_{\infty}^{n-1}\right)$. The injectivity of the right vertical arrow is the result of Lemma 3.3. Thus $P(\xi) \widehat{u}(\xi)=0$ implies that $\widehat{u}(\xi)$ is zero outside the origin. Thus $\operatorname{supp} \widehat{u}(\xi) \subset\{0\}$, hence $u(x)$ is entire infraexponential (if it is not zero).

REMARK 3.1. The above result is precise at $a=0$ because it then reduces to the case of Fourier hyperfunctions discussed in $\S 2$. But seemingly it may not be precise for other values of $a$. Especially, it is doubtful that the restriction $a \leq 1$ is really necessary to have a Liouville type theorem for some growth corresponding to the quasianalytic case, because if so, there would appear a kind of gap between $a=1$ and $a=\infty$. Notice, however, that in view of Seidenberg-Tarski's theorem, it is useless to consider the space of quasianalytic ultradistributions which are defined by weight functions finer than those of the type used here.

For the moment we have no idea how to prove a result in the opposite direction. The lack of such a tool also prevents the study of sharpness of the above condition.

REMARK 3.2. In Theorems 2.1 and 3.4 we assumed the growth condition also on the derivatives. This is to assume that $P(D) u=0$ holds in the respective space of generalized funcions on $\mathbb{D}^{n}$. We conjecture that as a matter of fact, the growth condition on the solution only would be enough. 
We expect that in some problems of asymptotic analysis at the top of corner or cone, as in Ziemian [Z], some similar phenomenon could be discovered separating the non-quasianalytic and quasianalytic cases, caused by the nasty elements supported by the top.

\section{References}

[G] A. Grothendieck, Topological Vector Spaces, Gordon and Breach, 1973.

[H1] L. Hörmander, Linear Partial Differential Operators, Springer, 1963.

[H2] -, Between distributions and hyperfunctions, Astérisque 131 (1985), 89-106.

[Kn1] A. Kaneko, On the structure of Fourier hyperfunctions, Proc. Japan Acad. 48 (1972), 651-653.

[Kn2] -, Introduction to Hyperfunctions, Univ. of Tokyo Press, 1980-1982 (in Japanese); English translation, Kluwer, 1988.

[Kn3] - Liouville type theorem for solutions of infra-exponential growth of linear partial differential equations with constant coefficients, Nat. Sci. Report Ochanomizu Univ. 49 (1998), 1-5.

[Kw] T. Kawai, On the theory of Fourier hyperfunctions and its applications to partial differential equations with constant coefficients, J. Fac. Sci. Univ. Tokyo Sect. 1A 17 (1970), 467-517.

[Km] H. Komatsu, Ultradistributions I, ibid. 20 (1973), 25-105.

[MY] M. Morimoto and K. Yoshino, Some examples of analytic functionals with carrier at the infinity, Proc. Japan Acad. 56 (1980), 357-361.

[P] V. P. Palamodov, From hyperfunctions to analytic functionals, Soviet Math. Dokl. 18 (1977), 975-979.

[PM] Y. S. Park and M. Morimoto, Fourier ultra-hyperfunctions in the Euclidean $n$-space, J. Fac. Sci. Univ. Tokyo Sect. 1A 20 (1973), 121-127.

[dR] J. W. de Roever, Hyperfunctional singular support of ultradistributions, ibid. 31 (1985), 585-631.

[SM] P. Sargos et M. Morimoto, Transformation des fonctionnelles analytiques à porteurs non compacts, Tokyo J. Math. 4 (1981), 457-492.

[S] L. Schwartz, Théorie des Distributions, new ed., Hermann, Paris, 1966.

[Z] B. Ziemian, The Mellin transformation and multidimensional generalized Taylor expansions of singular functions, J. Fac. Sci. Univ. Tokyo Sect. 1A 36 (1989), $263-295$

Department of Information Sciences

Ochanomizu University

2-1-1, Otsuka, Bunkyo-ku

Tokyo 112-8610, Japan

E-mail: kanenko@is.ocha.ac.jp 